\title{
HOT-ELECTRON TRANSPORT IN QUANTUM-DOT PHOTODETECTORS
}

\author{
L. H. CHIEN \\ EE Department, University at Buffalo, The State University of New York, 321 Bonner Hall, \\ Buffalo, NY 14260, USA ${ }^{*}$ \\ lchien2@buffalo.edu \\ A. SERGEEV \\ EE Department, University at Buffalo, The State University of New York, 321 Bonner Hall, \\ Buffalo, NY 14260, USA \\ asergeev@eng.buffalo.edu

\section{N. VAGIDOV} \\ EE Department, University at Buffalo, The State University of New York, 312D Bonner Hall, \\ Buffalo, NY 14260, USA \\ nizami@eng.buffalo.edu

\section{MITIN} \\ EE Department, University at Buffalo, The State University of New York, 332 Bonner Hall, \\ Buffalo, NY 14260, USA ${ }^{\S}$ \\ vmitin@buffalo.edu
}

\begin{abstract}
Employing Monte-Carlo simulations we investigate effects of an electric field on electron kinetics and transport in quantum-dot structures with potential barriers created around dots via intentional or unintentional doping. Results of our simulations demonstrate that the photoelectron capture is substantially enhanced in strong electric fields and this process has an exponential character. Detailed analysis shows that effects of the electric field on electron capture in the structures with barriers are not sensitive to the redistribution of electrons between valleys and these effects are not related to an increase of drift velocity. Most data find adequate explanation in the model of hotelectron transport in the potential relief of quantum dots. Electron kinetics controllable by potential barriers and an electric field may provide significant improvements in the photoconductive gain, detectivity, and responsivity of photodetectors.
\end{abstract}

Keywords: Quantum-dot photodetector; hot-electron transport; modulation doping; barriers, gain. 


\section{Introduction}

Numerous applications, ranging from flight control and night vision ${ }^{1}$ to commercial technologies in public safety, industry, and healthcare ${ }^{2}$ require sensitive scalable farinfrared sensors to employ in high-density focal plane arrays. Currently, marketable farinfrared technologies are mainly based on quantum-well infrared photodetectors (QWIPs). QWIPs are widely employed in various sensors and imaging devices operating at liquid nitrogen temperatures and below. ${ }^{3,4}$ At $77 \mathrm{~K}$, in the range near $\lambda \sim 10 \mu \mathrm{m}$ these sensors demonstrate the detectivity of $\sim 10^{10} \mathrm{cmHz}^{1 / 2} / \mathrm{W}$. The detectivity and responsivity drop drastically as the temperature increases to room temperature. Intensive investigations of electron transport in quantum-well structures have unambiguously shown that the high-temperature limitations of QWIP sensors are mainly caused by substantial reduction of photocarrier lifetime above the nitrogen temperatures. ${ }^{4}$ Fast picosecond photoelectron capture results in significant generation-recombination noise, which strongly decreases the detector sensitivity.

There is no doubt, a far-infrared detector operating at room temperature with reasonable sensitivity and responsivity would significantly increase the commercial market of IR technologies. Since the first publication, ${ }^{5}$ quantum-dot infrared photodectors (QDIPs) are being considered as the most promising candidate for achieving hightemperature operation. However, only after a decade of intensive studies, the roomtemperature far-infrared QDIPs have reached detectivities $\mathrm{D}^{*} \sim 10^{8} \mathrm{~cm} \mathrm{~Hz}^{1 / 2} / \mathrm{W}$ suitable for various commercial applications. ${ }^{6-8}$ Wide possibilities for manipulation with electron parameters in quantum dot-structures provide broad opportunities for further improving of QDIPs.

As we discussed above, suppression of photoelectron capture is the key issue for increasing sensitivity and responsivity of any semiconductor IR detector. Generally speaking, to suppress photoelectron capture one should separate the conducting electron states, which contribute to the electron transport, from the localized electron states, from which electrons can be excited by IR radiation. It is important to note that the electron kinetics in quantum-dot structures is substantially more manageable than that in the quantum-well superlattices. Geometry of photocarrier dot traps is restricted in all three dimensions. Therefore, the dots can be separated in the real space from the "conducting channels" by potential barriers. In our previous works, ${ }^{9-13}$ we suggested and studied various realizations of potential barriers and their effects on the detector performance. The barriers can be controlled over a wide range by varying host materials, doping level, and changing the characteristic lengths associated with the dot structure. The capture processes are determined by the electron diffusion in the field of potential barriers as well as intrinsic relaxation processes inside of the dots. The intra-dot relaxation combines an initial capture to the first bound state with subsequent relaxation to low energy levels. At room temperatures, the intra-dot processes are characterized by the picosecond rate, ${ }^{14,15}$ so without specially engineered barriers the photoelectron capture turns out to be too fast. $^{9-13}$ 
In the current paper, we investigate interplay of electron diffusion and intra-dot relaxation in electron transport through the quantum-dot structures with local potential barriers surrounding quantum dots. We associate the intra-dot relaxation with inelastic electron scattering on optical phonons and employ Monte-Carlo simulations to investigate effects of an electric field on electron capture. Our results show that the main features of electron kinetics in the quantum-dot structures can be understood in the model of hot-electron transport.

\section{Electron kinetics and detector performance}

The ultimate performance of a detector for specific applications is limited by the background limited performance (BLIP). In the BLIP regime, the average fluctuation of intrinsic "dark counts", $\sqrt{N_{d c}}$, should be smaller than the fluctuation of counts related to the background photon flux, $\eta \sqrt{N_{p h} A}$, where $\eta$ is the total quantum efficiency, $N_{p h}$ is the photon flux, and $A$ is the device area in the direction perpendicular to the flux. Thus, the background-limited sensitivity requires $N_{d c} / \eta^{2} \leq N_{p h} A$. In the semiconductor detectors, $N_{d c}$ is the generation rate of carriers to conducting states. It may be easily expressed through the concentration of the thermally generated carrier density, $n_{t h}$, and the carrier lifetime with respect to the capture to localized states, $\tau_{\text {capt }}: N_{d c}=n_{t h} V / \tau_{\text {capt }}$, where $V$ is the device volume. Combining the above equations, the BLIP requirement can be presented as

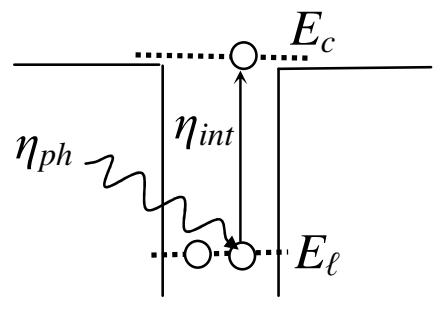

Fig. 1. Processes of photon absorption and electron transition with efficiencies $\eta_{p h}$ and $\eta_{i n t}$.

$$
G_{t h} \equiv \frac{n_{t h} d}{\tau_{c a p t} \eta^{2}} \leq N_{p h},
$$

where $d$ is the device thickness. The parameter $G_{t h}$, often referred to as the generation efficiency, is the basic characteristic, which is directly related with the device performance. Note, the analogous equation derived in Ref. 16, which is widely used in literature, is different from Eq. (1) by a factor of $\eta$, i.e. it has $\eta$ (not $\eta^{2}$ ) in the denominator. Such an equation ignores the fluctuation character of $N_{d c}$ and $N_{p h}$, and, therefore, is incorrect. The quantum efficiency, $\eta=\eta_{p h} \eta_{i n t}$, includes the coupling constant with electromagnetic radiation, $\eta_{p h}$, and the intrinsic quantum efficiency, $\eta_{\text {int }}$.

In thermodynamic equilibrium the thermally generated carrier density may be expressed in terms of the equilibrium electron concentration in localized states in dots, $n_{0}$,

$$
n_{t h}=n_{0} g_{s} \exp \left(-\frac{E_{c}-E_{l}}{k T}\right),
$$


where $E_{c}$ and $E_{\ell}$ are the energy of conducting and localized electron states correspondingly, and $g_{s}$ is the factor of electron statistics: $g_{s}=1$ for degenerate electrons in localized states and $g_{s}=k T / \varepsilon_{F}$ in the opposite case ( $\varepsilon_{F}$ is the electron Fermi energy). Thus, $G_{t h}$ may be presented as

$$
G_{t h}=\frac{g_{s} n_{0} d}{\tau_{c a p t} \eta_{p h}^{2} \eta_{\text {int }}^{2}} \exp \left(-\frac{E_{c}-E_{l}}{k T}\right) .
$$

Taking into account that $G_{t h}$ is directly related to the detectivity of the device,

$$
D^{*}=\left(h v \sqrt{2 G_{t h}}\right)^{-1}
$$

we see that $G_{t h}$ (Eq. (3)) is a figure of merit, which provides a simplistic method for analysis of available devices and discussion of possible ways to improve them.

For an optimally designed device, the system of energy levels should correspond to the electromagnetic radiation to be detected, i.e. the energy of the quantum $h v$ should just slightly exceed $E_{c}-E_{\ell}$. The exponential factor in Eq. (3) describes strong dependence of optimal QDIP performance on the radiation wavelength.

Modern room temperature operating QDIPs have $n_{0} d \sim 10^{15} / \mathrm{cm}^{2}, g_{\mathrm{s}} \sim 1$, and $\eta$ up to $0.3-0.4 .^{14,15}$ The carrier lifetime, $\tau_{\text {capt }}$, at room temperatures is of the order of 1 ps or even shorter. Substituting these parameters in Eqs. (3) and (4), we evaluate the detectivity for $\lambda=10 \mu \mathrm{m}$ as $D^{*} \approx 0.5 \times 10^{8} \mathrm{cmHz}^{1 / 2} / \mathrm{W}$, which is in agreement with the very recent achievements in QDIP performance. It means that the potential of the current QDIP design is practically exhausted and further improvements will require new ideas and changes in the detector design.

According to Eq. (3), the capture time of photoelectrons $\tau_{\text {capt }}$ is an important parameter for the detector performance. Besides the reduction of the generationrecombination noise, slow photoelectron capture also improves the photoconductive gain, which is defined as

$$
g=\tau_{\text {capt }} / \tau_{t r}
$$

where $\tau_{t r}$ is the electron transit time, i.e. time that an electron spends in the device moving from the emitter to the collector. Increase of the gain does not directly improve the intrinsic sensitivity (detectivity) of the device, but increases the responsivity which is proportional to the gain. High responsivity provides an easy matching of the device with its readout and decreases the noise of the readout amplifier in the complete system.

To suppress photoelectron capture one should separate the conducting electron states that provide electron transport from localized electron states which are excited by IR radiation. The capture processes can be restricted by specially-engineered potential barriers around dots. ${ }^{9-13}$ For example, such barriers can be obtained in structures with homogeneous doping of the inter-dot space. ${ }^{9}$ In this case, electrons from dopants populate quantum dots and create depletion areas around the dots. The barrier potential is created by electrons bounded in quantum dots and ionized impurities in the depletion areas. In 
this case the barrier potential is determined by the geometrical parameters of the structure as well as the doping level. Potential barriers of another form may be created, if electrons populating the dots were taken from the specific areas located relatively far from the dots.

\section{Photoelectron capture: Quasi-equilibrium distribution}

As in our previous works, ${ }^{9-13}$ we consider a quantum-dot structure with potential barriers around dots. In what follows the detailed form of the barriers is not critical. The only important assumption we accept is that the probability of tunnelling processes is small compared with the capture probability via thermo-excitation above the potential barrier. For example, for dots with $a \sim 10 \mathrm{~nm}$ the potential that satisfies the above condition can be created by the homogeneous doping of the inter-dot space. ${ }^{9}$ Without tunneling processes, the photoelectron capture rate,

$$
\tau_{\text {capt }}^{-1}=N_{d} \sigma \tilde{v}
$$

is given by the equation for the trapping cross-section, ${ }^{11}$

$$
\begin{gathered}
\sigma=\pi \alpha a^{2} \exp \left(-\frac{e V_{m}}{k T}\right)\left(1+\frac{3}{4} \frac{\alpha a}{\ell} F(V)\right)^{-1}, \\
F=a \exp \left(-\frac{e V_{m}}{k T}\right) \int_{a}^{b} \frac{d r}{r^{2}} \exp \left(\frac{e V(r)}{k T}\right),
\end{gathered}
$$

where $\tilde{v}$ is the electron thermal velocity, $N_{d}$ is the concentration of quantum dots, $a$ is the radius of the dot, $b$ is the inter-dot distance, $\ell$ is the electron mean free path with respect to elastic electron scattering, $\alpha$ is the probability for an electron at $r \leq a$ to be captured by the quantum dot, and $V_{m}$ is the maximum value of the potential barrier, i.e. $V_{m}=V(a)$. The equations above are analogous to formulas obtained by Pekar for electron trapping by attractive impurity traps. ${ }^{17}$

We would like to emphasize that Eqs. (7) and (8) are valid for any relation between $\ell$, $a$, and $\alpha a$ as well as for wide variety of potentials. To compare these results with conclusions of Ref. 14, let us consider the flat potential $V=0$. In this case,

$$
\sigma=\pi \alpha a^{2}\left[1+\frac{3}{4} \frac{\alpha a}{\ell}\right]^{-1}
$$

Following to Ref. 14, we accept that the inelastic intra-dot relaxation processes are described by the relaxation time $\tau_{\varepsilon}^{\prime}$. In this case, the coefficient $\alpha$ can be evaluated as $\alpha \approx a / \ell_{\varepsilon}^{\prime}$, where $\ell_{\varepsilon}^{\prime}=\widetilde{v}^{\prime} \tau_{\varepsilon}^{\prime} ; \widetilde{v}^{\prime}$ is the electron thermal velocity in the dot. Then, if $a^{2}<<\ell \ell_{\varepsilon}^{\prime}$, we obtain the capture rate

$$
\frac{1}{\tau_{\text {capt }}}=\pi N_{d} a^{3} \frac{\tilde{v}}{\tilde{v}^{\prime}} \frac{1}{\tau_{\varepsilon}^{\prime}}
$$


In the opposite case, $a^{2}>>\ell \ell_{\varepsilon}^{\prime}$, the capture rate is independent of the coefficient $\alpha$ and is given by

$$
\tau_{\text {capt }}^{-1}=4 \pi N_{d} D a
$$

where $D=\tilde{v} \ell / 3$ is the diffusion coefficient. Both limiting cases are in agreement with results of Ref. 14, which were obtained in other formalism. ${ }^{18}$ Note, that the second term in the square brackets in Eq. (9) describes the reduction of the carrier concentration near the dot due to the capture processes. As we discussed, this effect becomes important in the electron capture, if $\ell<\alpha a$, and results in Eq. (11). In the opposite case, the electron concentration is practically homogeneous in space and carrier capture is determined by Eq. (10).

Returning to the capture processes in the presence of potential barriers, we should note that the second term in the brackets in Eq. (7) also describes the reduction of the carrier concentration near the dot. Due to repulsive potential barriers this effect is increased by a factor of $F(V)$ given by Eq. (8). Comparing this result with electron capture on repulsive impurity traps, one can associate $F(V)$ with the Sommerfeld factor, which shows the reduction of carrier density (electron wavefunction) near the trap. If local reduction of carrier density is negligible, the capture rate is

$$
\frac{1}{\tau_{\text {capt }}}=\pi N_{d} a^{3} \frac{\tilde{v}}{\tilde{v}^{\prime}} \frac{1}{\tau_{\varepsilon}^{\prime}} \exp \left(-\frac{e V_{m}}{k T}\right),
$$

where the exponential factor describes the effect of potential barriers on capture processes.

Summarising this section, we should note that, while the above formalism is general enough, it is applicable only for quasi-equilibrium electron distributions, i.e. in small electric fields. At the same time, optimal regimes for operating of semiconductor detectors are always achieved in strong fields, which generate nonequilibrium carrier distributions. In the next section we investigate effect of an electric field on capture processes.

\section{Carrier capture in an electric field}

Monte-Carlo simulation is an effective tool for investigations of capture processes in external fields. Our program includes all basic scattering processes, including electron scattering on acoustic, polar optical, and intervalley phonons. Our modeling includes electrons in $\Gamma$-, $\mathrm{L}-$, and $\mathrm{X}$-valleys and takes into account redistribution of carriers between valleys. With this Monte-Carlo program we simulate transport of three-dimensional electrons in GaAs matrix with InGaAs dots. We assume that the dots

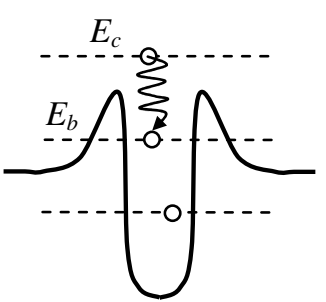

Fig. 2. The carrier capture as a result of electronphonon scattering. 


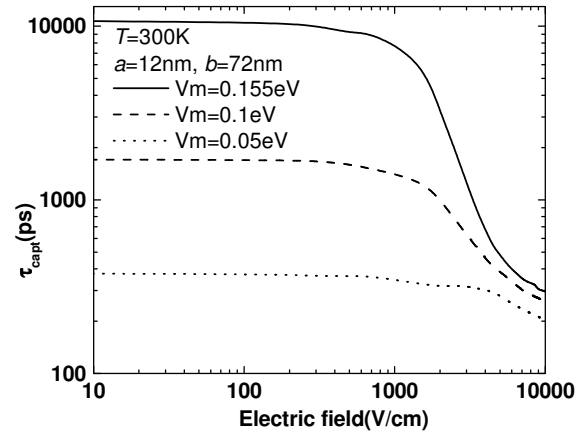

Fig. 3. Carrier capture time as a function of the electric field at various potential barriers.

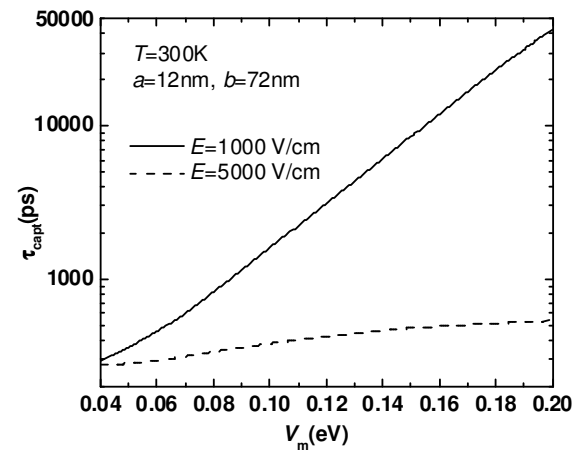

Fig. 4. Capture time as a function of the barrier height at various electric fields.

are arranged in a regular lattice with the inter-dot distance of $b$. Then, the dot concentration $N_{d}$ is given by $1 / b^{3}$. As it was discussed in the previous section, we accept that the intra-dot relaxation processes are described by the relaxation time $\tau_{\varepsilon}^{\prime}$, which is associated with inelastic electron-phonon scattering in the dot area (see Fig. 2). Thus, here we consider the carrier capture process as a specific scattering process: (i) which is limited in space by the dot volume, (ii) in which a carrier transits from a conducting state with the energy $E_{c}$ above the potential barrier to a bound state $E_{b}$, which is below the potential barrier. In other words, we will assume that from the bound state a carrier will relax to the deep dot states faster than it could return back to the conducting state.

In the model above we investigate the dependence of the carrier capture on the electric field at various values of the potential barrier. As seen in Fig. 3, the capture time is practically independent on the electric field up to the critical field $F_{c}$, which is of the order of $10^{3} \mathrm{~V} / \mathrm{cm}$, and than substantially decreases with the field increase. Figure 4 shows the dependence of the capture time on the value of potential barrier. Note, that significant changes in this dependence appear only in electric fields larger than $1 \mathrm{kV} / \mathrm{cm}$. Comparing these data with the results of the previous work, ${ }^{12,13}$ where we did not specify the intra-dot processes and accepted $\alpha=1$, we can make an important conclusion. While the absolute values of the capture rate depend strongly on intra-dot processes, the dependencies of $\tau_{\text {capt }}$ on the electric field are modified just slightly in a wide range of variations of $\alpha$. Thus, the characteristic value of the electric field $F_{c}$ is mainly determined by the carrier transport in the matrix, and it is insensitive to intra-dot processes.

To compare the data of our simulations with analytical results, we investigated the dependence of the capture time on the dot radius in the electric field. As seen in Fig. 5, the product of $\tau_{\text {capt }}$ and $a^{3}$ is a universal function of the electric field $F$. Taking into account that at room temperature the electron mean free path $\ell$ is significantly larger than the dot radius, we expect that without an electric field the capture rate is proportional to $a^{3}$, as it is described by Eq. (12). Our Monte-Carlo modeling demonstrates that this is 


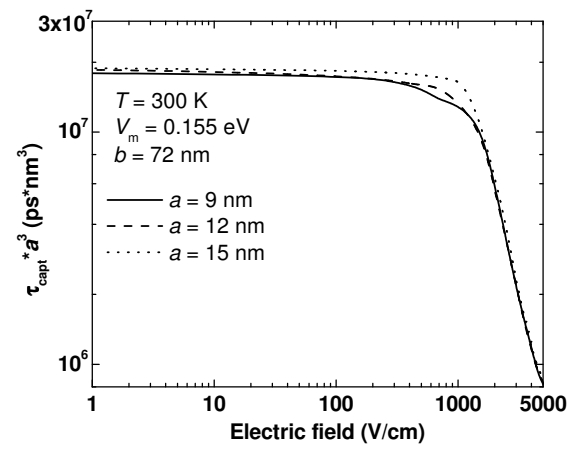

Fig. 5. Product of $\tau_{\text {capt }}$ and $a^{3}$ as a function of the electric field.

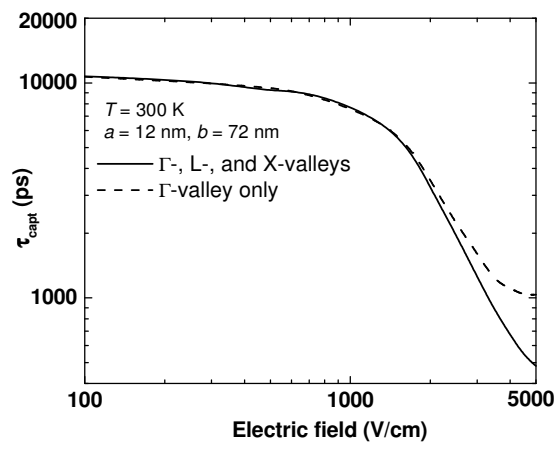

Fig. 6. Contributions of L-, $X-$ and $\Gamma$-valleys: Solid line - all valleys; dashed line capture from $\mathrm{L}$ - and $\mathrm{X}$-valleys is prohibited $\left(\alpha_{L, X}=0\right)$.

also valid in the electric fields, including a wide range of strong electric fields that substantially modify the capture rate.

In a recent paper, ${ }^{19}$ it has been suggested that the conducting and localized electron states can be effectively separated in the $k$-space as well as in the real space. GaAs has a complex band structure, which consists of $\Gamma$-, L-, and X-valleys. In equilibrium electrons mainly populate $\Gamma$ - valley. In substantial electric fields, electrons can transfer from $\Gamma$ - to L- and X-valleys. The upper valleys are characterized by high values of electron masses and, therefore, by high densities of states. For this reason, in high electric fields the transfer to higher valleys is strongly enhanced. After electrons repopulate L- and Xvalleys, the electron capture rate from these states into $\Gamma$-like localized states in the dots is substantially smaller than that from $\Gamma$ -

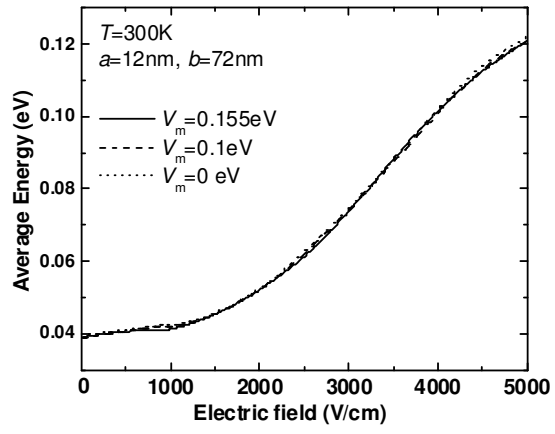

Fig. 7. Average electron energy $\overline{\mathcal{E}}$ as a function of the electric field. valley, and therefore, separation in the $k$ space may result in significant reduction of the cross-section for capture processes.

To check this hypothesis, we calculated the capture time averaged over electrons in $\Gamma$ - valley and compared it with the result for all electrons. As seen in Fig. 6, the effect of upper valleys in the carrier capture becomes important only at very high electric fields $(\sim 3 \mathrm{kV} / \mathrm{cm})$, which are substantially larger than the characteristic fields $F_{c}$ related to potential barriers.

Finally, let us try to analyze the modeling data in terms of electron heating. The dependence of the average electron energy $\overline{\mathcal{E}}$ on the electric field $F$ is shown in Fig. 7. 

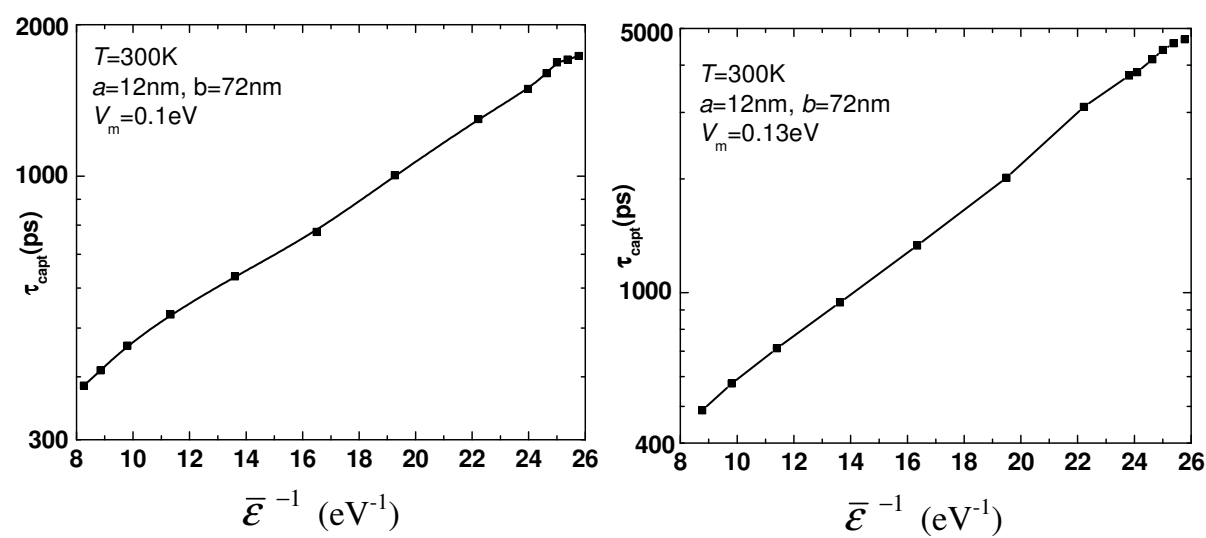

Fig. 8. The capture time as a function of inverse average electron energy attained in the electric field.

As expected, potential barriers do not change the average energy attained in the electric field. In Fig. 8, we present the logarithmic dependence of the capture time on the inverse value of $\bar{\varepsilon}$. As seen, for various potential barriers $\log \tau_{\text {capt }}$ is proportional to $1 / \bar{\varepsilon}$, i.e. we find that $\tau_{\text {capt }}$ is proportional to $\exp (1 / \bar{\varepsilon})$. Therefore, we may conclude that the carrier capture in the electric field can be described by Eq. (12), where the thermal energy $k T$ is replaced by a factor of $\sim 2 \bar{\varepsilon} / 3$. Thus, the carrier capture is well described by the model of electron heating.

In summary, a wide range of novel properties may be realized through the manipulation of dots and potential barriers created by selective doping in quantum-dot structures. The potential barriers around the dots may be used to separate the localized intra-dot electron states from the conducting states in the matrix and, in this way, to control all electron processes. In structures with the barriers, electron kinetics and transport in the electric field is well described by the model of electron heating. Thus, the barriers strongly suppress the capture processes until the average energy of carriers becomes comparable with the barrier height. Controllable kinetics is expected to provide a significant increase in the photoconductive gain, device detectivity, and responsivity. Manageable kinetics will also allow one to employ quantum-dot sensors as an adaptive detector with changing parameters.

\section{Acknowledgements}

The research was supported by AFOSR and NYSTAR grants. 


\section{References}

1. M. Z. Tidrrow, Quantum Sensing and Nanophotonic Devices II 5732, 217, Proceed. SPIE edited by M. Razeghi and G. J. Brown (2005).

2. A. Rogalski, Progress in Quantum Electronics 27, 59 (2003).

3. S. D. Gunapala, S. V. Bandara, J. K. Liu et al., Semicond. Sci. Technol. 20, 473 (2005).

4. B. F. Levine, J. Appl. Phys. 74, R1 (1993).

5. V. Ryzhii, Semicond. Sci. Technol. 11, 759 (1996).

6. P. Bhattacharya, X. H. Su, S. Chakrabarti et al., Appl. Phys. Lett. 86, 191106 (2005).

7. S. Tsao, H. Lim, W. Zhang, and M. Razeghi, Appl. Phys. Lett. 90, 201109 (2007).

8. H. Lim, S. Tsao, W. Zhang, and M. Razeghi, Appl. Phys. Lett. 90, 131112 (2007).

9. V. V. Mitin, V. I. Pipa, A. V. Sergeev et al., Infrared Phys. and Technol. 42, 467 (2001).

10. V. Ryzhii, I. Khmyrova, V. Mitin et al., Appl. Phys. Lett. 78, 3523 (2001).

11. A. Sergeev, V. Mitin, and M. Stroscio, Physica B 316-317, 369 (2002).

12. V. Mitin, N. Vagidov, and A. Sergeev, Phys. Stat. Sol. C 3, 4013 (2006).

13. N. Vagidov, A. Sergeev, and V. Mitin, Int. J. High Speed Electronics and Systems 17, 585 (2007).

14. H. Lim, B. Movaghar, S. Tsao et al., Phys. Rev. B 74, 205321 (2006).

15. M. Razeghi, H. Lim, S. Tsao et al., Nanotechnology 16, 219 (2005).

16. J. Phillips, J. Appl. Phys. 91, 4590 (2002).

17. S. I. Pekar, Zh. Eksp.Teor. Fiz. 20, 267 (1950).

18. K. K. Ghosh, L.-H. Zhao, and D. L. Huber, Phys. Rev. B 25, 3851 (1982).

19. M. R. Matthews, R. J. Steed, and M. D. Frogley, Appl. Phys. Lett. 90, 103519 (2007). 